\title{
Dalla didattica in presenza alla didattica a distanza: la favola di Natale di Guareschi
}

\author{
di Silvia Pascale
}

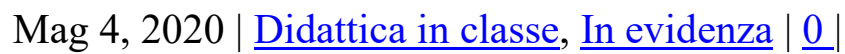

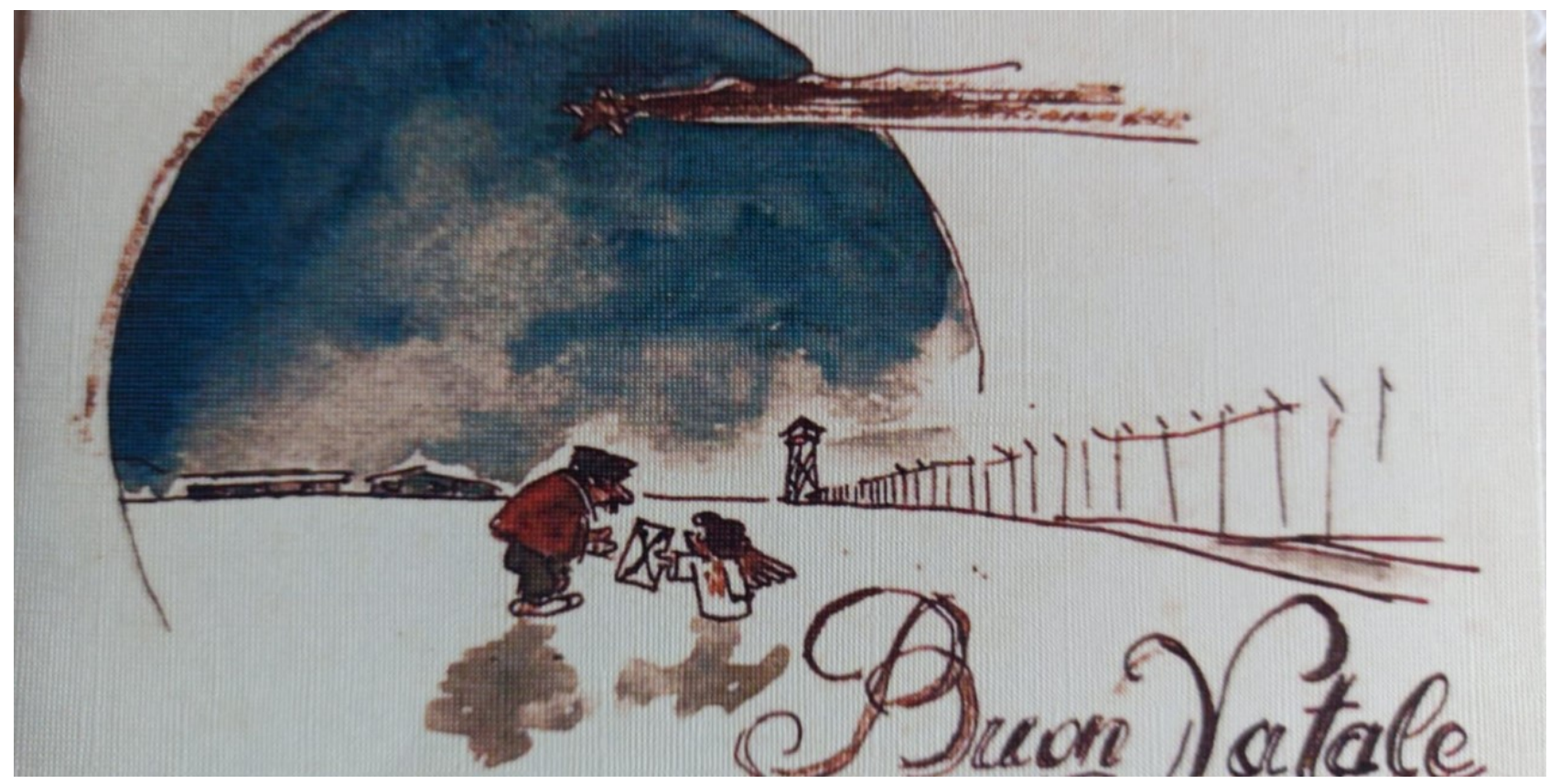

Sandbostel dicembre 1944 disegno di Giovannino Guareschi mentre riceve dall'angelo postino gli auguri di Natale dai suoi cari (archivio Guareschi, riproduzione autorizzata dagli Eredi di Giovannino Guareschi.)

\begin{abstract}
La Favola di Natale di Guareschi è un testo teatrale scritto e rappresentato in una baracca del campo di concentramento per Internati Militari Italiani (IMI) di Sandbostel nel 1944. Attraverso un percorso laboratoriale articolato, nel primo quadrimestre di quest'anno due classi dell' IC4 Stefanini di Treviso hanno studiato la deportazione degli IMI con fonti scritte e fotografiche, per poi arrivare alla lettura dell'opera di Guareschi: l'analisi del testo era stata progettata per essere accompagnata anche allo studio delle parti musicali per la messa in scena finale che avrebbe dovuto avvenire il 7 maggio 2020. A fine febbraio la sospensione delle lezioni in presenza, per via dell'epidemia di COVID-19, ha portato a dovere modificare il prodotto finale del lavoro di ricerca: i ragazzi sono diventati protagonisti attivi non più della rappresentazione, ma del volume di cui la docente coinvolta è anche curatrice per la prossima pubblicazione.
\end{abstract}

\section{PREMESSA}

Questo progetto è stato presentato per l'a.s. 2019/2020 all'IC4 Stefanini di Treviso dove insegno Lettere in 2 classi seconde. Il lavoro è stato pensato per poter essere replicato e è situato all'interno di un percorso di formazione sulla Memoria per docenti intitolato "Ripensare la Memoria" e che prevede un focus sull'internamento militare italiano, ossia sui militari deportati nei campi del Terzo Reich dopo 1'8 settembre 1943.[1] 
Il progetto risponde principalmente alla necessità riscontrata nell' Istituto coinvolto di sviluppare competenze in materia di cittadinanza attiva e democratica e comportamenti responsabili ispirati alla conoscenza e al rispetto della legalità attraverso lo studio approfondito e articolato de $L a$ Favola di Natale di Guareschi.

\section{OBIETTIVI DIDATTICI}

Gli obiettivi didattici che si sono perseguiti in questo lavoro sono:

- favorire lo sviluppo di riflessioni personali attraverso lavori di gruppo in modalità peer to peer e di cooperative learning;

- utilizzare la lettura del testo teatrale di Giovannino Guareschi, scritto nel campo di concentramento di Sandbostel, per riflettere sull'internamento militare italiano.

Era previsto che La Favola di Natale sarebbe stata messa in scena all'Auditorium dell'Istituto il 7 maggio 2020, alla presenza dell'Associazione Nazionale ex Internati nei Lager nazisti (ANEI), proprio dai ragazzi che si sarebbero cimentati nello studio e nell'analisi storica e con la supervisione di Alberto Guareschi, figlio dell'autore. Contemporaneamente sarebbe stato anche presentato il volume di approfondimento storico su opera e autore. Concepito all'origine del percorso e da me curato, prevedeva una serie di saggi di taglio prettamente storico: un'introduzione del presidente nazionale dell'ANEI, Orlando Materassi, un saggio storico sugli avvenimenti che portarono all'8 settembre 1943 del Generale Maurizio Lenzi, presidente dell'ANEI di Padova e del Museo Nazionale dell'Internamento, due saggi miei sulla figura di Guareschi e i Lager dove è stato internato, oltre all'adattamento del testo de La Favola di Natale di Marco Crepet e a un saggio sulla musica dell'opera curato da Gioele Gusberti.

\section{PERCHE' GUARESCHI? PERCHE' "LA FAVOLA DI NATALE"}

La favola di Natale è un'opera letteraria dello scrittore e giornalista Giovannino Guareschi scritta nel dicembre del 1944 durante il periodo di prigionia a Sandbostel e venne raccontata per la prima volta la sera della Vigilia di Natale dello stesso anno nella sua baracca nel campo di prigionia.

La scelta del testo La Favola di Natale ${ }^{[2]}$ di Giovannino Guareschi ${ }^{[3]}$ è stata effettuata sulla base della valenza interdisciplinare dell'opera: teatro, musica, storia, letteratura, arte.

Si tratta, infatti, di un testo scritto per essere recitato, illustrato dall'autore stesso e musicato dall'amico e compagno di prigionia Arturo Coppola. Quest'ultimo, una volta rientrato in Italia, ha stabilito la sua residenza a Treviso e ha insegnato musica proprio nell'istituto scolastico coinvolto.

La favola narra di un bambino di nome Albertino, che è il figlio dell'autore, della sua nonnina, del suo cagnolino Flick, di una lucciola e del loro incredibile onirico viaggio verso il campo di concentramento in cui si trova il padre. Durante il viaggio essi incontrano funghi parlanti, cornacchie canterine, oggetti animati, angeli e tante altre stranissime creature.

Nella premessa della favola Guareschi indica come proprie muse ispiratrici Freddo, Fame e Nostalgia e racconta che Arturo Coppola, che musicò la fiaba, diresse l'orchestra e il coro degli internati la sera di Natale del 1944. Ricorda l'autore in un'intervista rilasciata dopo la guerra: «Per l'umidità i violini si scollavano, perdevano il manico e le voci faticavano a uscire da quella fame vestita di stracci e di freddo». Eppure la fiaba di Albertino restituì un sorriso ai prigionieri.

L'autore stesso così esordisce: 
Questa favola è nata in un campo di concentramento del Nord-ovest germanico, nel dicembre del 1944, e le Muse che l'ispirarono si chiamavano Freddo, Fame e Nostalgia. Questa favola io la scrissi rannicchiato in un "castello" biposto, e sopra la mia testa c'era la fabbrica della melodia. Io mandavo su da Coppola versi di canzoni nudi e infreddoliti, e Coppola me li rimandava giù rivestiti di musica soffice e calda come lana d'Angora. [...] Ma la sera della Vigilia, nella squallida baracca del "Teatro", zeppa di gente malinconica, io lessi le favole e l'orchestra, il coro e $i$ cantanti la commentarono egregiamente, e il "rumorista" diede vita ai passaggi più movimentati.

[...] Io vi racconterò una favola e voi la racconterete al vento di questa sera, e il vento la racconterà ai vostri bambini. E anche alle mamme e alle nonne dei vostri bambini, perché è la nostra favola: la favola malinconica d'ognuno di noi. Io, la sera della Vigilia del '44, conclusi con queste parole la premessa: ma il vento avrà sentito? $O$, se ha sentito, sarà riuscito poi a superare $i$ baluardi della censura? O, lungo la strada, avrà perso qualche periodo? Ci si può fidare del vento in un affare cosi delicato?

Un'autentica parabola, la definirei, sull'amore, sulla famiglia e sull'importanza dei valori: il viaggio onirico di Albertino, che colpisce anche per i caratteri fiabeschi e per le trovate poetiche, è un manifesto all'amore fra gli esseri umani, non solo in tempo di guerra (tema ben evidente e contestualizzato), ma anche in tempo di pace.

Le personificazioni che si diversificano tra comparse buone e cattive, spingono i ragazzi ad un'ulteriore riflessione sulla bontà delle nostre intenzioni più nascoste.

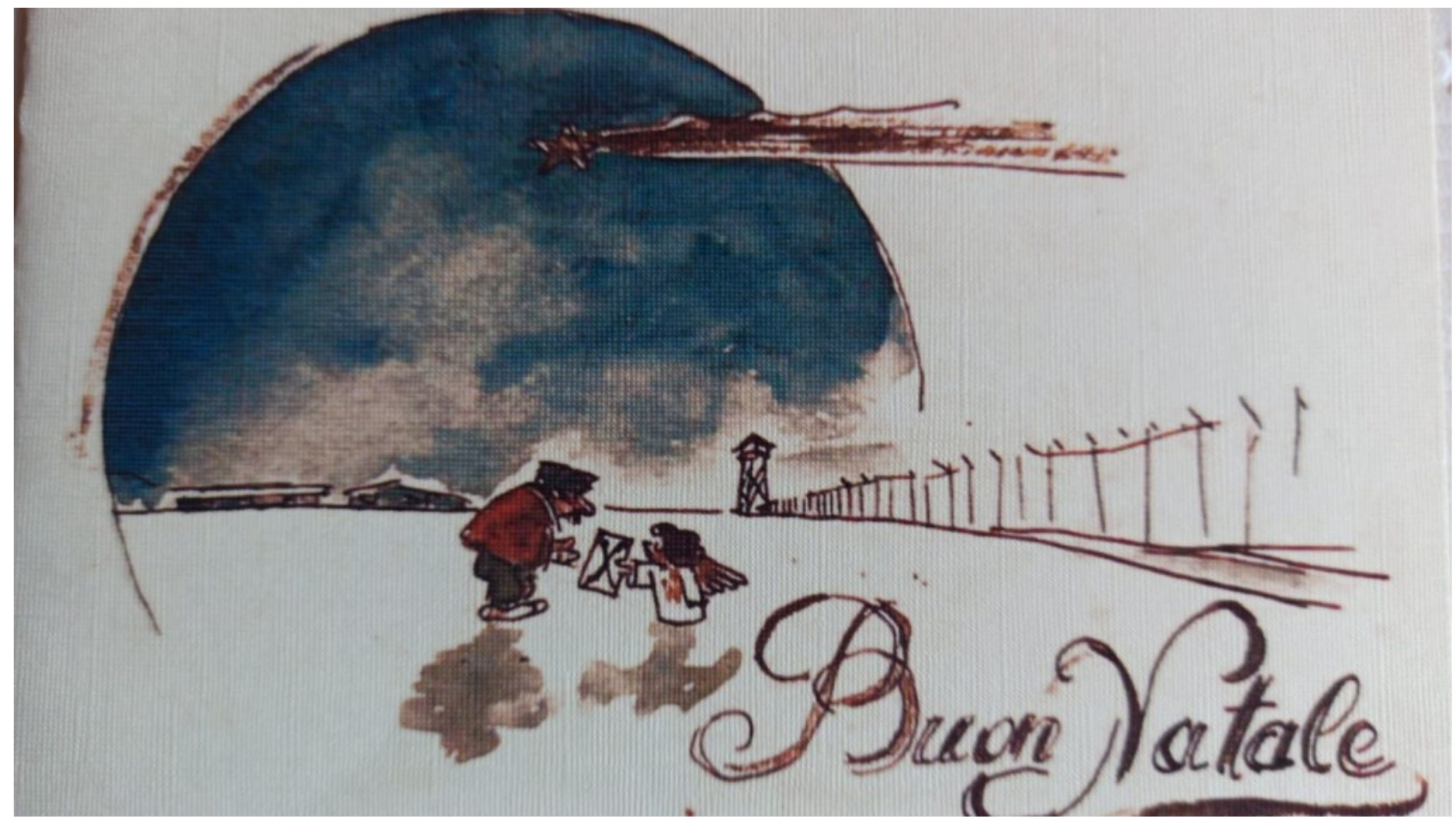

Sandbostel dicembre 1944 disegno di Giovannino Guareschi mentre riceve dall'angelo postino gli auguri di Natale dai suoi cari (archivio Guareschi, riproduzione autorizzata dagli Eredi di Giovannino Guareschi.)

\section{ASPETTO INTERDISCIPLINARE}


Il progetto è finalizzato a potenziare la comunicazione in Lingua 1 attraverso un approfondimento culturale delle tematiche del Novecento riguardo ai genocidi, al razzismo, alla violenza e al dovere della Memoria.

Nella progettazione è stata prevista anche la conoscenza del linguaggio teatrale, della modalità di trasposizione di temi in immagini e della trasversalità dei linguaggi espressivi; nonché l'apprendimento di una dizione corretta e la memorizzazione di testi con l'arricchimento lessicale.

L'Istituto coinvolto ha un coro e un'orchestra composti dagli allievi della scuola e quindi il punto di forza del progetto è consistito anche nello studio e nell'esecuzione delle parti cantate e suonate dai ragazzi. E fondamentale la consapevolezza dell'importanza dell'espressione creativa di idee, esperienze ed emozioni in un'ampia varietà di mezzi di comunicazione, quali la musica e il canto.

Tutto ciò è possibile attraverso il potenziamento delle metodologie laboratoriali e delle attività di laboratorio. Il progetto si è configurato fin da subito come attività laboratoriale in continuo divenire, e ha permesso la sperimentazione di varie tecniche e metodologie didattiche. I ragazzi hanno svolto le attività previste dal progetto in un'ora alla settimana (l'ora di approfondimento di Lettere) per la durata di tutto l'anno scolastico.

\section{IL LABORATORIO DI STORIA}

Dopo un'introduzione storica all'argomento legata in particolare alla deportazione dei militari italiani nei lager tedeschi dopo 1'8 settembre 1943, è stato avviato con le classi un percorso attraverso le fonti con il Laboratorio di Storia.

È importante, prima di procedere alla presentazione delle fonti, spiegare il valore che esse rappresentano per la tematica: perché un argomento interessi e attivi volontà di comprensione e di ricerca non basta che sia proposto dall'insegnante, è importante che il ragazzo riconosca il significato della conoscenza che gli si chiede di apprendere.

Ne consegue che è necessario elaborare una strategia dello stimolo (un problema da chiarire o da risolvere, una domanda cui trovare risposta, un prodotto da realizzare ecc.) tale da coinvolgere lo studente. Nel nostro caso era la messa in scena de La Favola di Natale di Guareschi.

Utilizzo spesso, quando possibile, il Laboratorio di Storia per offrire agli studenti la capacità di leggere il passato attraverso il concreto gesto storiografico, al fine di raggiungere una più completa consapevolezza del presente. L'apprendimento della storia dovrebbe avvenire attraverso calibrate operazioni di tipo storiografico, offrendo materiali e strumenti per rendere gli allievi non passivi destinatari, ma costruttori di percorsi di storia. Ciò comporta la necessità di lavorare in piccoli gruppi in modalità peer to peer possibilmente, entro uno spazio dedicato e con una precisa frequenza settimanale. Il processo di peer education[4] parte dal confronto tra punti di vista diversi; attraverso lo scambio di idee e la condivisione delle esperienze avviene una comunicazione circolare da cui scaturiscono percorsi di apprendimento. L'insegnamento reciproco consente agli studenti di accrescere e perfezionare le proprie conoscenze, i metodi di studio e la capacità di problem solving. In questa strategia didattica lo studente tutor viene valorizzato e responsabilizzato da questo ruolo mentre gli altri studenti traggono vantaggio dal lavorare in un ambiente protetto tra pari.

La scelta delle fonti è stata varia per permettere agli alunni di ampliare le loro conoscenze su di un periodo storico non trattato nei libri di testo scolastici e per sperimentare varie tipologie di materiali: 
1. pagine di diario di un militare italiano internato: in particolare la pagina iniziale nella quale vengono descritti gli avvenimenti immediatamente successivi all' 8 settembre '43; la pagina dove viene descritta la schedatura nel Lager come IMI; due pagine dedicate al lavoro forzato (in questo caso in una miniera di carbone vicino a Düsseldorf);

2. cartoline spedite dai prigionieri dai Lager di internamento;

3. foto da Archivi storici (Croce Rossa Internazionale e Bundesarchiv);

4. tessere di riconoscimento di lavoratori coatti;

5. Personalkarte, esempio di schedatura all'arrivo nello Stalag di un internato militare.

Le scritture diaristiche, come anche le lettere, hanno un rapporto preciso con il tempo: i diari veri e propri, le lettere, procedono per frammenti, senza un disegno, prive di una dimensione prospettica. Nel diario, in particolare, si riproduce o si riflette, più o meno inconsciamente, la molteplicità $o$ l'occasionalità dell'esistenza. Ma le caratteristiche del diario (e delle lettere) sono anche i suoi limiti. Il testimone racconta ciò che vede, ciò che osserva dal suo punto di vista e spesso non sa o non capisce il contesto. E da qui nasce poi con gli alunni il confronto tra ciò che il testimone scrive e quello che troviamo sui saggi di storia.

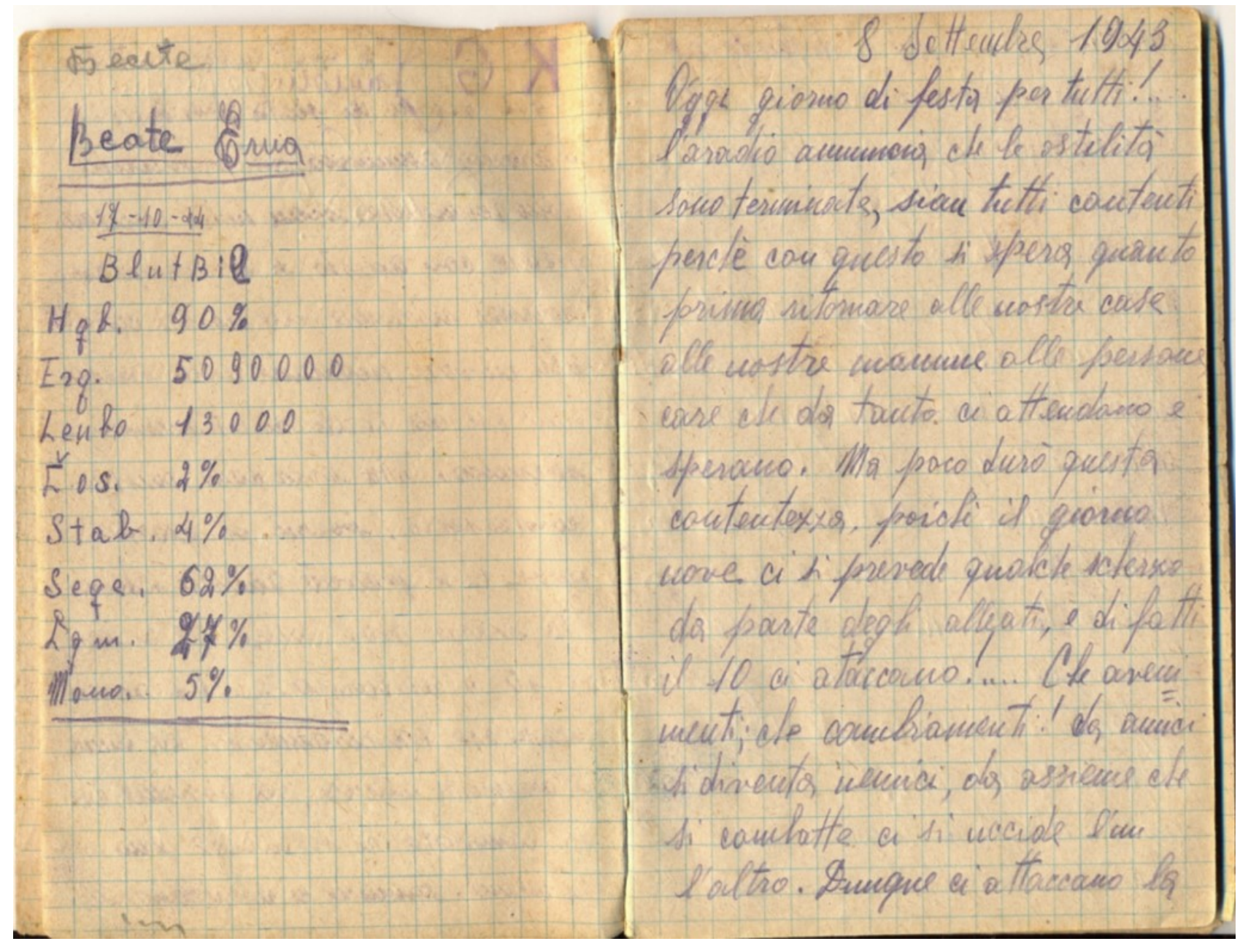

Pagina di diario di Alfredo Zaros (Pascale 2017) 


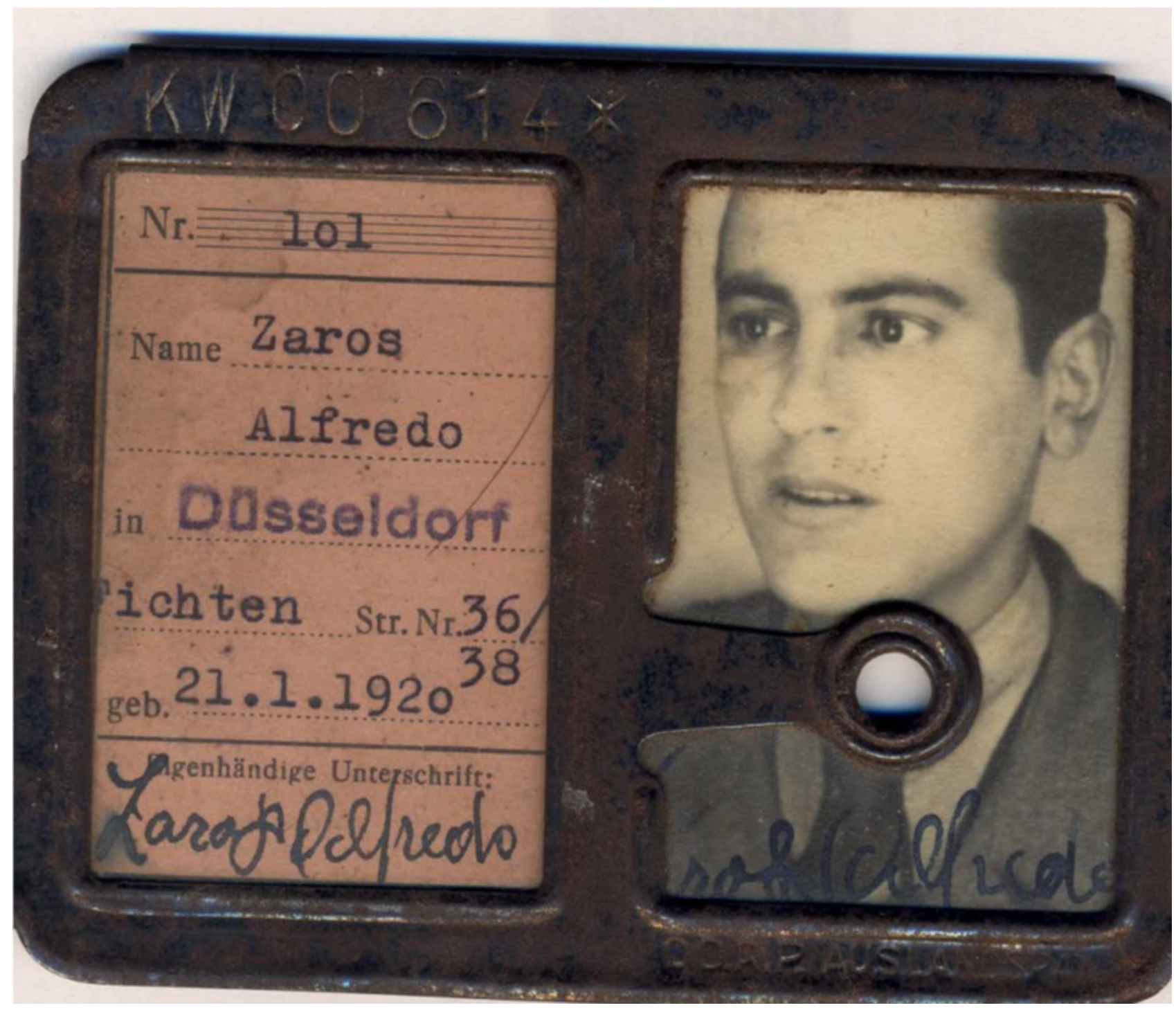

Tesserino da lavoratore coatto di Alfredo Zaros (Pascale 2017) 


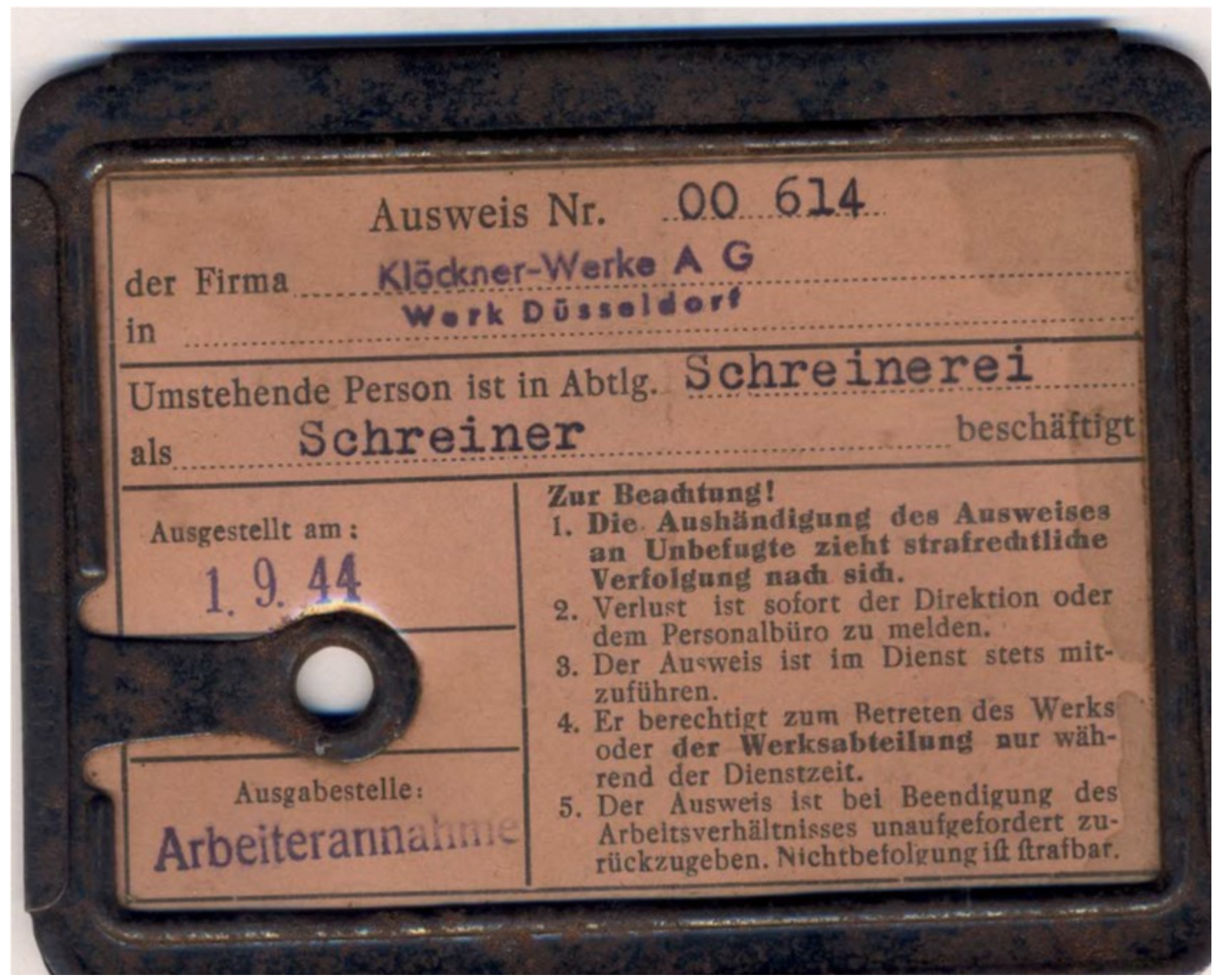

Tesserino da lavoratore coatto di Alfredo Zaros (Pascale 2017) 


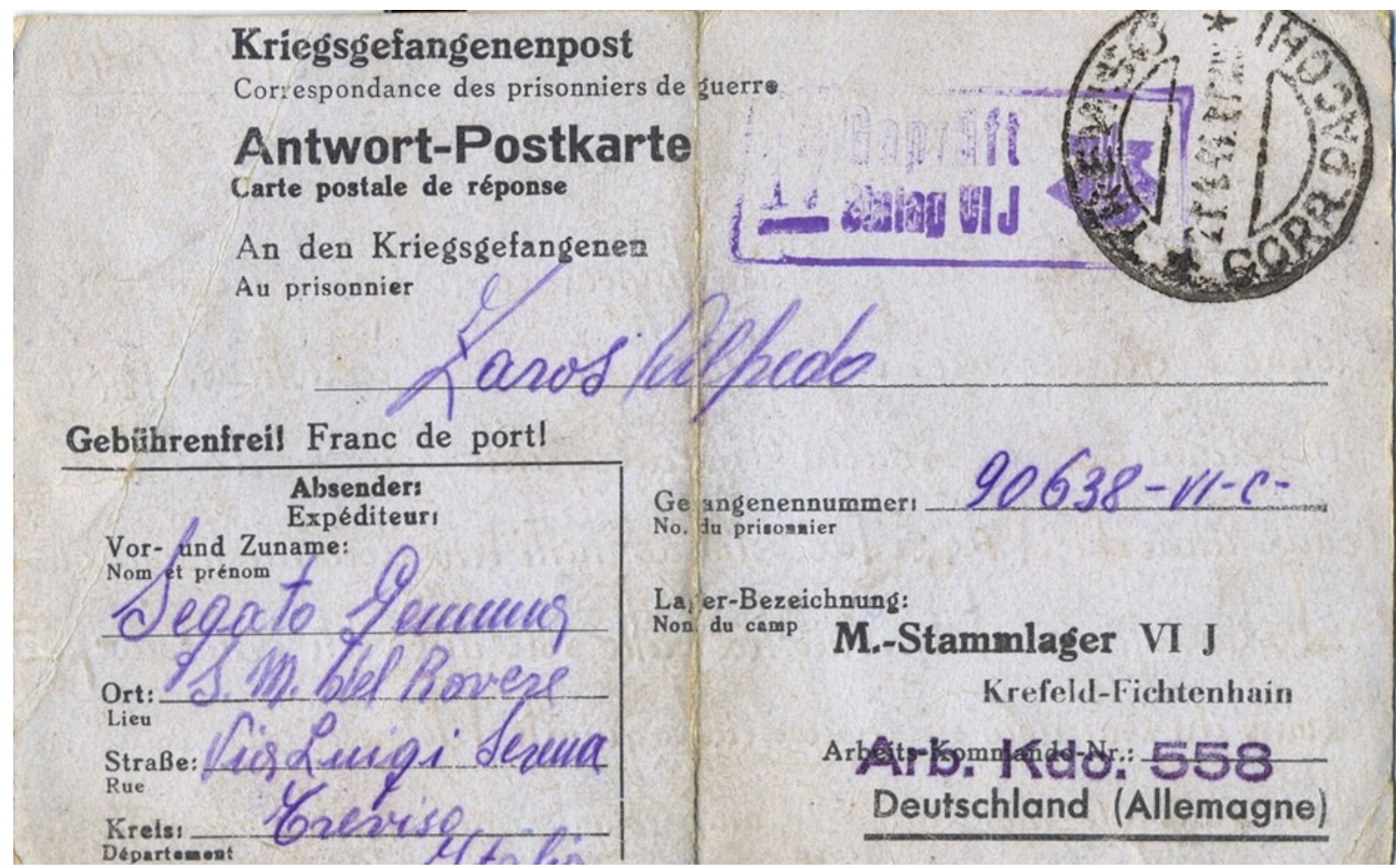

Cartolina IMI Alfredo Zaros (Pascale 2017)

Si può dire che le attività di laboratorio hanno il loro punto di forza e sono caratterizzate da questi elementi:

- ruolo attivo dello studente;

- svolgimento di un'attività di una certa durata e finalizzata alla realizzazione di un prodotto;

- analisi di documenti da cui trarre informazioni;

- rielaborazione delle stesse;

- collaborazione con altri compagni nelle diverse fasi del lavoro.

Attraverso le fonti proposte gli studenti passano dall'osservazione alla conoscenza interpretando poi il significato del documento stesso, proponendo anche delle ipotesi. 


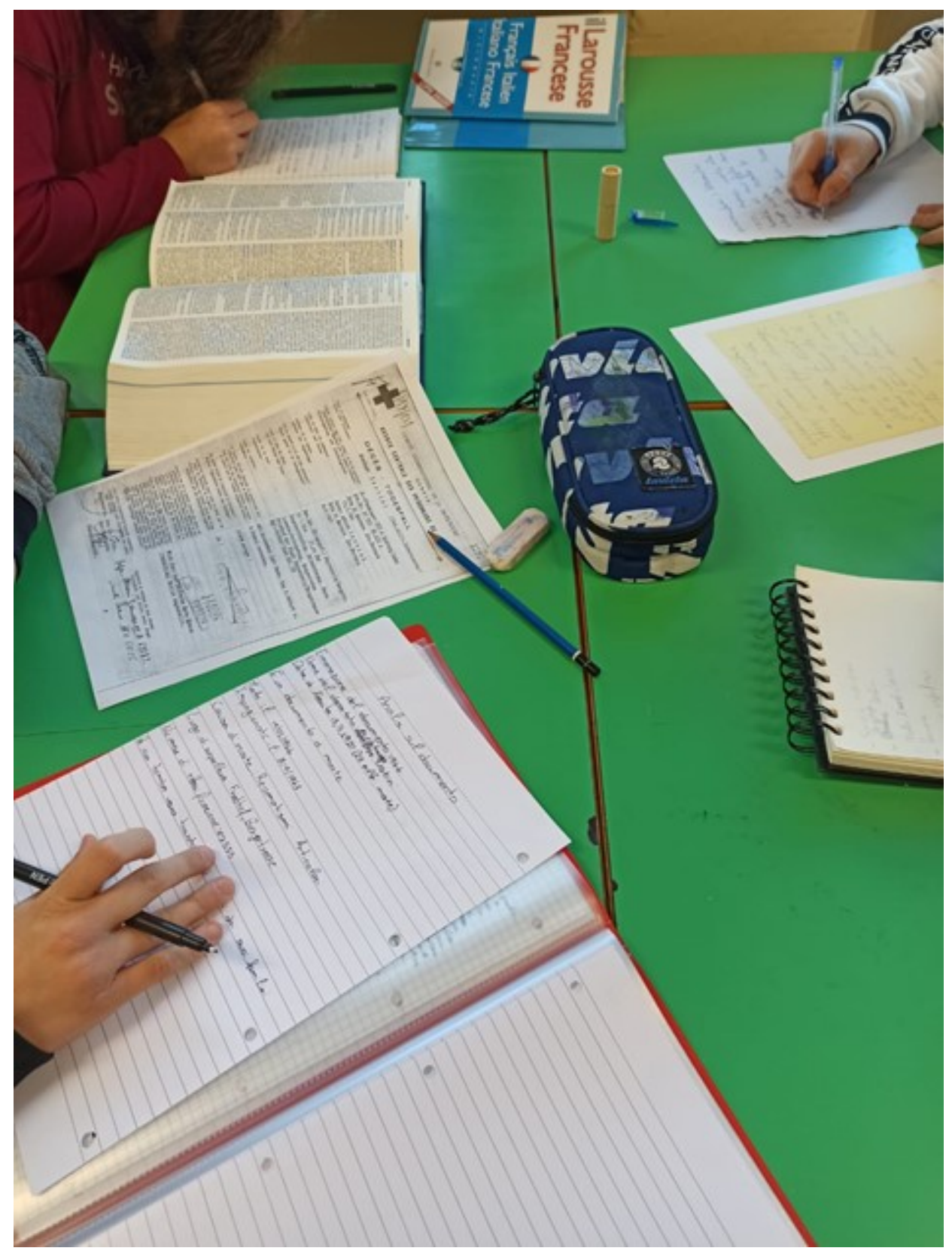

Lavoro a piccoli gruppi sui documenti di prigionia di IMI (foto Silvia Pascale)

Lo scopo del laboratorio è duplice: da un lato fornire i primi rudimenti circa gli strumenti della ricerca condotta a partire da una fonte indicata dal docente, e dall'altro insegnare allo studente come si elaborano in forma scritta i risultati della ricerca.

Al termine di questi incontri settimanali gli studenti acquisiscono la conoscenza di alcune tipologie di fonti per lo studio degli IMI e dei principali strumenti di studio. Dopo essersi orientati nella ricerca e decodifica dei documenti, utilizzano gli strumenti linguistici (proprietà di linguaggio, corretto lessico specifico) adeguati a esprimere nella forma scritta, in modo chiaro ed efficace, gli esiti della ricerca condotta sotto la guida del docente.

Queste competenze vengono acquisite tramite un confronto diretto e continuativo con il docente in aula; la discussione intorno alle fonti proposte costituirà inoltre un momento di riflessione critica $\mathrm{e}$ di apprendimento metodologico. 
La prima parte del laboratorio viene utilizzata per spiegare il motivo e l'obiettivo del lavoro di ricerca, come si compiono le indagini bibliografiche e archivistiche, come si seleziona il materiale e dove lo si reperisce, che cosa significa scrivere i risultati della ricerca e come si fa.

Una fonte cui i ragazzi vanno preparati accuratamente è quella fotografica/iconografica: non è sufficiente che gli studenti diano una rapida occhiata all'immagine. Devono invece essere in grado di descriverla in modo dettagliato e sistematico e saper dare un nome preciso agli oggetti ed alle persone rappresentati. Il che vuol dire padroneggiare da un lato l'abilità linguistica (scritta e orale) della descrizione e dall'altro disporre di un ampio bagaglio di informazioni sull'immagine che deve essere "letta" e sul contesto storico. Solo a questo punto si può provare a decodificare con una certa profondità l'immagine, stimolando poi in classe eventuali discussioni.

Successivamente lo spunto è stato quello di confrontare l'immagine del campo di concentramento durante la Seconda Guerra e lo stesso luogo adesso.

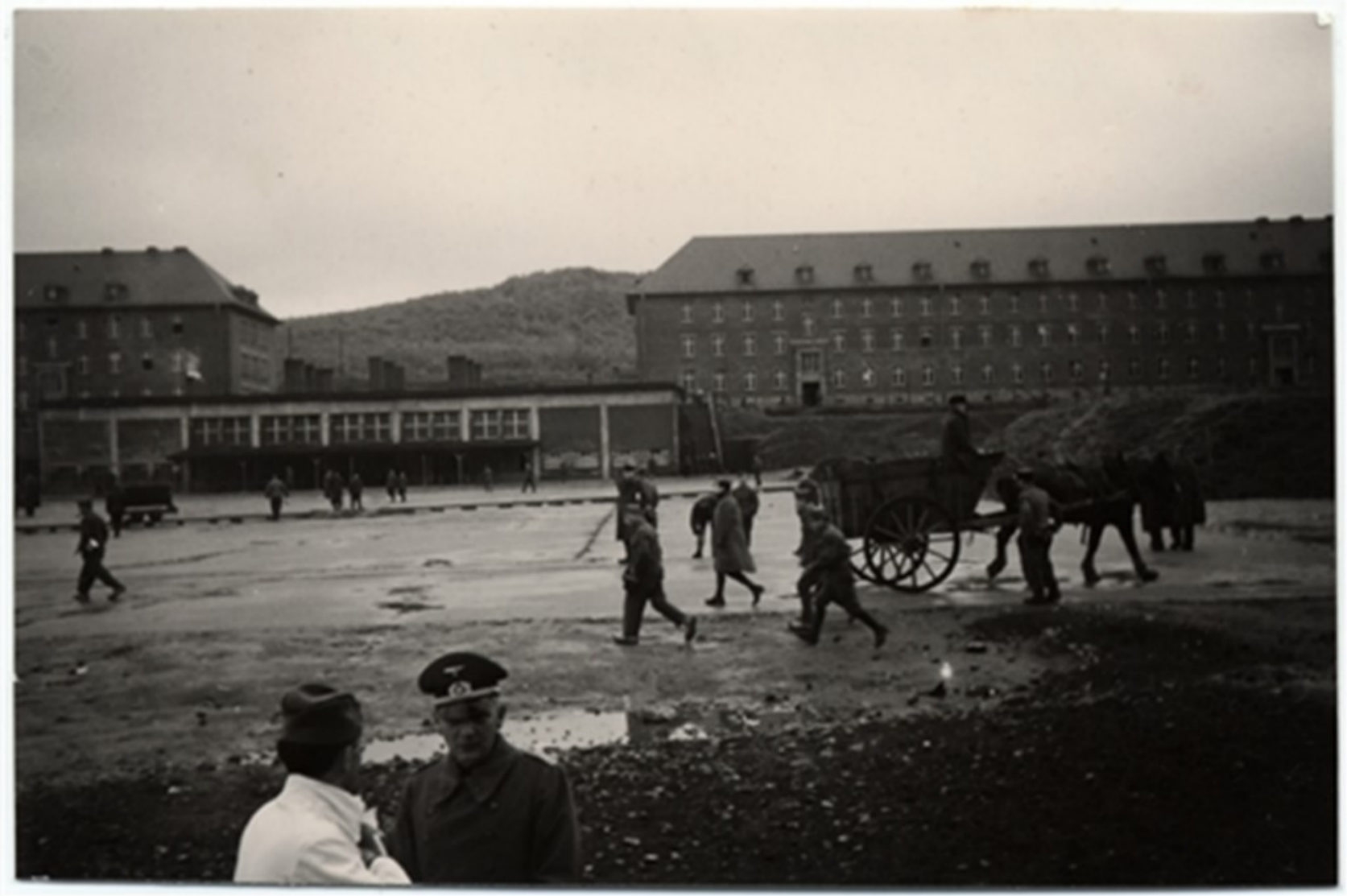

Stalag VI A, Hemer: Archivio Storico Croce Rossa Internazionale ICRC VP-HIST-03104-28 


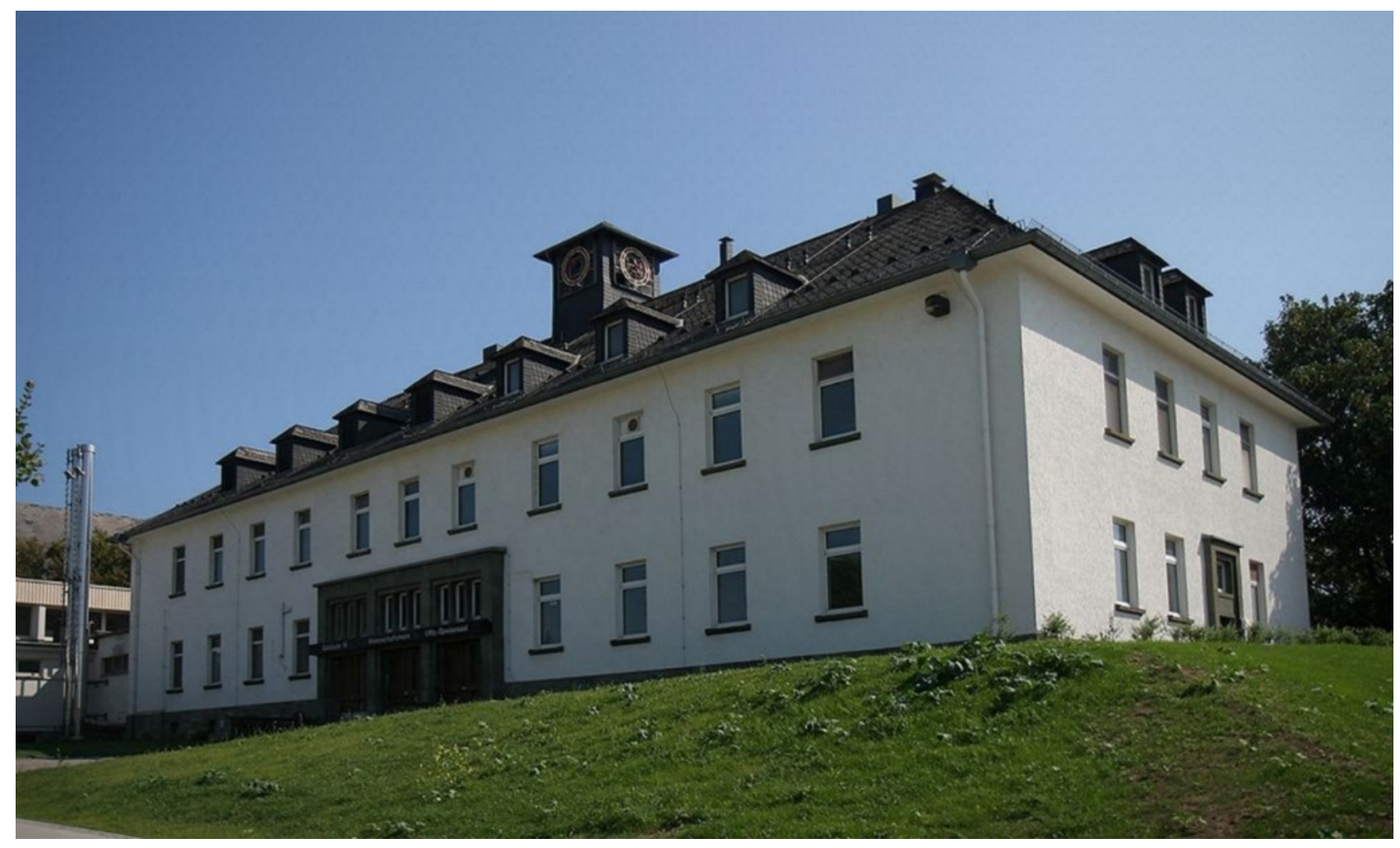

Stalag VI A, Hemer: Archivio Storico Croce Rossa Internazionale ICRC VP-HIST-03104-28

La seconda parte del laboratorio, invece, vede l'esposizione dei lavori svolti dagli studenti in gruppo. In questo passaggio gli studenti descrivono ciò che si può trarre come interpretazione dalle diverse fonti da loro utilizzate riguardo all'argomento ed analizzano come possono differire queste fonti tra di loro. Individuano come sono state costruite le memorie legate all'argomento scelto e mettono a confronto la memoria individuale e quella collettiva.

Questa parte della metodologia costituisce una sfida in quanto gli studenti ora lavorano con il concetto di memoria a livello pratico, non soltanto teorico.

L'obiettivo è quello di incentivare le discussioni e le presentazioni delle idee proprie degli studenti. Per iniziare il loro lavoro, gli studenti devono compilare un riassunto di ciò che hanno imparato utilizzando le domande seguenti:

1. Quale periodo storico/argomento stai analizzando?

2. Quale fonte storica hai analizzato?

3. Per la decodifica della fonte/documento che strumenti ti sono serviti?

4. Riassumi cosa dice la fonte in merito all'argomento.

\section{TEMPI DEL PROGETTO ORIGINARI PRIMA DEL COVID 19}

I QUADRIMESTRE (ottobre - gennaio 1 - 2 ore alla settimana): RIPENSARE LA MEMORIA

\section{APPROFONDIMENTI}

- Approfondimento sulle tematiche della deportazione.

- La deportazione dei militari italiani dopo l'8 settembre 1943. 
- Laboratorio sui documenti di alcuni IMI (Internati Militari Italiani) in modalità piccoli gruppi.

- Testimonianza della prof.ssa Silvia Pascale pronipote di un IMI morto in lager.

- Incontro con il presidente nazionale ANEI, Orlando Materassi, figlio di un internato.

Sicuramente di forte impatto emotivo sono stati i due incontri di inizio febbraio legati alla testimonianza familiare di internamento: ascoltare il racconto di deportazione e di vita nel campo, costretto al lavoro coatto, di Elio Materassi, attraverso le parole del figlio, ha indubbiamente avuto una valenza educativa enorme. La storia è sicuramente anche storia di testimonianze, semplici come quella di Elio, ma con una forte connotazione educativa.

Le testimonianze di IMI attraverso i documenti oppure ascoltando figli o nipoti di internati, sono uguali e diverse tra loro, perché tutte hanno una comune storia ed ognuna ha l'originalità personale, ma sono importantissime per comprendere il valore della scelta dei 650.000 militari italiani fatti prigionieri dall'esercito tedesco dopo l'armistizio dell'8 settembre 1943: preferirono il volontario internamento pur di non schierarsi dalla parte dei nazifascisti. 


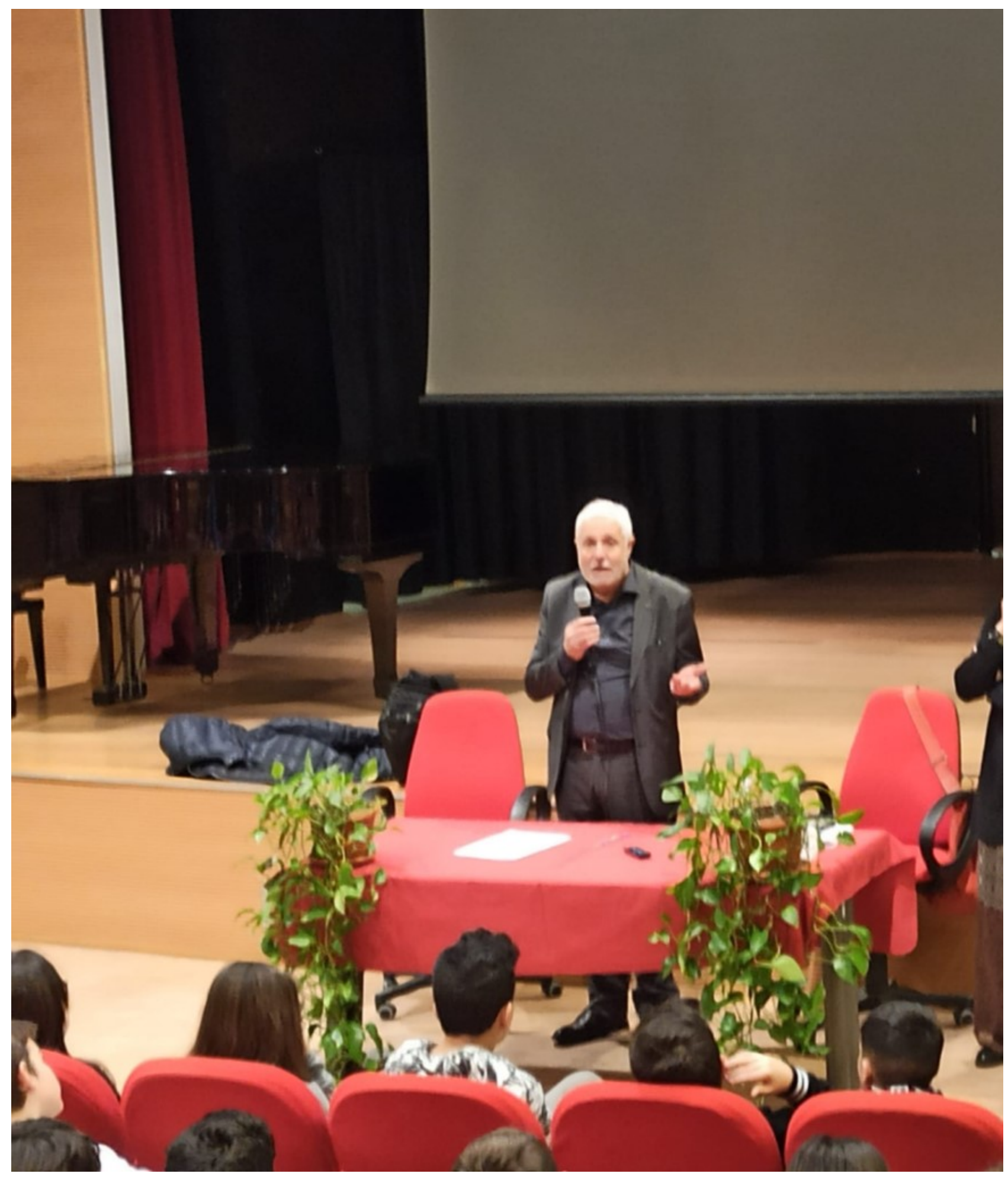

Incontro con Orlando Materassi, presidente nazionale ANEI (foto Silvia Pascale)

Il loro "NO" ripetuto più volte, fu causa di sofferenze personali: fame, freddo, affetti negati, violenze e tanti altri supplizi, ma la loro lotta senz'armi contribuì alla liberazione e rendere dignità a un'Italia per venti anni soggiogata dalla dittatura fascista e distrutta dalla guerra voluta dai regimi nazista e fascista con la complicità della Casa Reale. 50.000 di loro troveranno la morte nei Lager a causa di sfinimento, malattie, per i bombardamenti o per essere stati uccisi singolarmente o in gruppo dai nazisti. 
Gli studenti, nel percorso del primo quadrimestre, hanno imparato che anche l'internamento fu una delle tante storie di deportazioni durante la Seconda guerra mondiale, con la particolarità della volontaria scelta di restare nei Lager a loro destinati - che si chiamavano Stalag per sottufficiali e soldati di truppa e Oflag per ufficiali - pur di non aderire al nazifascismo. Nei campi di Internamento per Militari Italiani capitava spesso arrivassero funzionari del Reich oppure della Repubblica Sociale Italiana per chiedere ai prigionieri di arruolarsi con i nazifascisti in cambio di vitto e alloggio adeguato e il rimpatrio. All'interno dei lager una modesta parte degli IMI opterà e firmerà l'adesione al nazifascismo con il sogno di rientrare in Italia, sperando di avere cibo migliore e abbondante: una volta firmata l'adesione potevano nascere scontri verbali o fisici con coloro che non avevano aderito.

Un mondo complesso e difficile che i testimoni contribuiscono a ricostruire. Nel suo interessantissimo volume Annette Wieviorka individua diverse figure di testimone che si sono succedute nel tempo:

il testimone oculare, depositario della storia a "futura memoria", di solito scomparso con il mondo stesso che ha descritto, lasciando la sua testimonianza in un testo scritto; il testimone sopravvissuto che vive i problemi del reinserimento e della dissimulazione; il testimone discendente dello scampato che racconta non solo il passato ma anche la narrazione di una nuova vita. [5]

Per questo motivo alla fine del percorso sui documenti si è rivelato di particolare interesse per i ragazzi ascoltare la mia storia familiare e quella di Orlando Materassi.

\section{QUADRIMESTRE (febbraio - aprile): LA FAVOLA DI NATALE}

\section{APPROFONDIMENTI}

- Lettura del testo.

- Divisione e assegnazione delle parti recitate e musicate.

- 7 maggio: rappresentazione teatrale.

\section{FEBBRAIO 2020: SOSPENSIONE della DIDATTICA IN PRESENZA}

La sospensione dell'attività didattica in presenza e l'impossibilità di effettuare le prove ha reso impossibile proseguire con il progetto originario. Ho comunicato ai ragazzi collegati in videolezione che purtroppo il lavoro sarebbe slittato al successivo anno scolastico: appena data la notizia ho visto lo sconforto e la delusione, ma subito uno degli studenti ha lanciato la proposta di inserire dei propri lavori nel volume dedicato che stava per essere pubblicato. E subito è stato un susseguirsi di emozioni...

"Prof. Io sono brava a disegnare"... "Prof io vorrei scrivere una poesia!"

Ecco riaccendersi la gioia di fare insieme, anche se distanti, di condividere un lavoro, di confrontarsi e discutere come facciamo sempre in classe.

"Prof., Guareschi ha scritto quest'opera in campo di concentramento ed è stato isolato dai suoi affetti per due anni; anche noi siamo isolati dai nostri amici, dai nostri cugini."

"Prof. io mi sento come Giovannino: non posso più andare dai nonni... Dai prof. vedrà che bravi che siamo!!!!” 
Ecco che ci siamo messi all'opera: abbiamo riletto insieme la favola in video-lezione, ognuno dei ragazzi si è soffermato su dei personaggi che più l'avevano colpito; piano piano sono nate idee per $\mathrm{i}$ disegni; uno studente ha preparato degli origami e c'è chi ha dipinto con la pianta dei piedi. E ora, con il supporto anche dei genitori che sostengono questo lavoro e di un'amica in particolare, Michela Bayou che ha coordinato la parte grafica, si sta procedendo verso la bozza finale del testo che vede impegnati gli studenti delle mie due classi seconde. Anche i ragazzini certificati DSA, altri con il docente di sostegno, alcuni stranieri che rischiano di perdersi senza la normale attività scolastica, partecipano sapendo che il loro contributo sarà considerato perché tutti avranno il loro spazio.

È un lavoro, quello dello studio della deportazione dei militari italiani dopo l'8 settembre nei lager del Terzo Reich in cui credo molto: credo che sia fondamentale passare ai nostri ragazzi questa pagina di storia che non compare, se non a volte per brevi cenni, nei nostri manuali scolastici.

Così Alberto, il figlio di Guareschi, mi scrive al primo invio dei materiali:

\section{Cara Silvia,}

grazie di cuore per avermi inviato in anteprima i disegni e la commovente poesia che alcuni suoi alunni hanno dedicato a mio padre e alla sua Favola di Natale. Una favola - le cui muse ispiratrici furono «fame, freddo e nostalgia» - nata per consolare gli internati militari nei Lager tedeschi lontani dai loro cari ed è riuscita a rasserenarli tenendoli aggrappati alla vita e alla speranza di poterli rivedere. È confortante sapere che questa favola riesce a coinvolgere emotivamente anche questa nuovissima generazione...

Un augurio per una felice conclusione in "videoconferenza". 


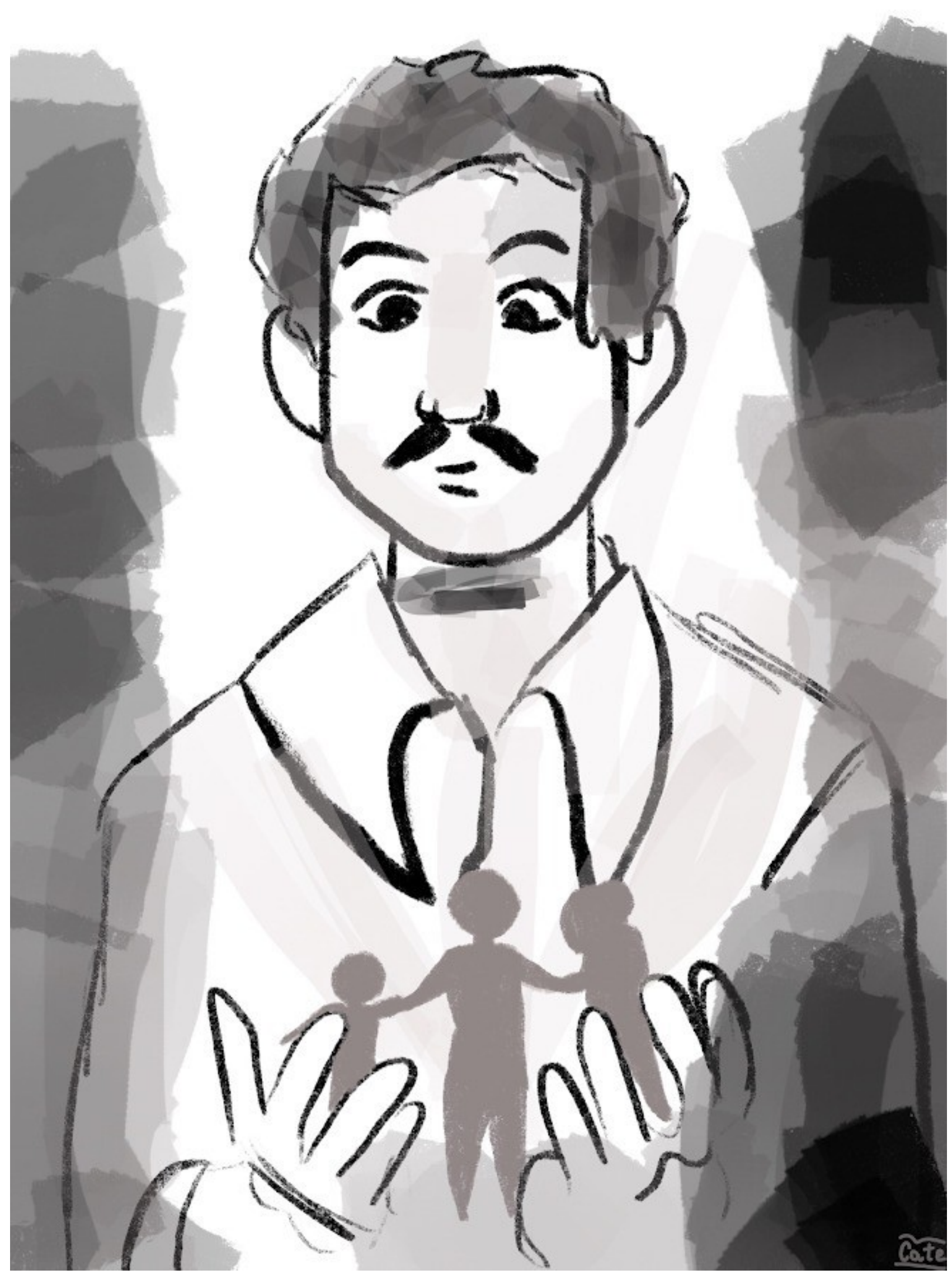


Disegno di Caterina A. 2 H: Guareschi pensa a chi lo aspetta a casa, la moglie e i due figli, Alberto e Carlotta.

\section{MODIFICA AL PROGETTO INIZIALE}

La cadenza settimanale con i ragazzi è rimasta confermata, anche se sono cambiate le modalità: ci colleghiamo con Meet della piattaforma G-Suite for Education che avevamo già in dotazione come Istituto. Una volta alla settimana, quindi, lavoriamo sulla Memoria, non più però una classe alla volta, ma contemporaneamente, perché con Meet c'è la possibilità di collegare i ragazzi di tutte e due le classi. I lavori che i ragazzi producono, vengono caricati sul drive di Classroom, un sistema che permette di archiviare qualsiasi file in modo sicuro e illimitato, condividendo in modo rapido e invitando anche i compagni e i colleghi di sostegno a visualizzare ed eventualmente commentare.

Il volume originariamente progettato è stato ripensato e adattato al nuovo obiettivo che prevede il coinvolgimento editoriale delle classi. In questo senso è stata aggiunta la parte didattica: la premessa di una carissima amica docente con cui condivido tematiche legate alle deportazioni nazifasciste, Pamela Stranieri; una riflessione sul laboratorio di storia con Francesca Piaser; la spiegazione del percorso didattico in modalità DAD e la produzione degli alunni.

Ecco quindi nascere questo volume scritto veramente "a cento mani" con i ragazzi che hanno voluto comunque continuare a credere nel progetto e che anzi lo hanno arricchito.

È un contributo importantissimo affinché altri ragazzi e ragazze nati nel terzo millennio abbiano la conoscenza e consapevolezza di costruire il loro futuro facendo tesoro di cosa sia la mancanza di libertà, di democrazia e di pace.

La voce degli internati, che affiora dalle pagine di diari o lettere, ci impone di farci carico di queste sofferenze e ci spinge a diventare a nostra volta testimoni attivi: è la via per contrastare qualsiasi forma di negazionismo e per far sì che questo periodo della storia venga studiato dai nostri ragazzi.

Per diventare poi testimoni bisogna andare oltre l'aspetto meramente cognitivo: la memoria prevede anche l'emozione, la condivisione di un'esperienza, il pathos.

Solo così si potrà cercare di avere un passaggio di testimone vero ed efficace: infatti non soltanto intelligenza e razionalità aiutano l'apprendimento, ma anche le emozioni.

L'attività grafica in particolare ha avuto un ruolo importante: i disegni sono uno strumento per restituire un punto di vista autonomo sull'argomento trattato per creare un ponte fra pensieri ed emozioni.

Come anteprima di tale ricca produzione propongo qui alcuni dei lavori dei ragazzi, alternandoli tra testi, disegni e poesia da cui si può percepire l'emozione. Tutti questi lavori saranno inseriti nel volume che uscirà a breve intitolato Guareschi e il Natale nel Lager, Ciesse Edizioni, Padova 2020.

Nicolò descrive lui stesso il suo elaborato:

Questo disegno che ho realizzato simboleggia un campo di concentramento. Un luogo dove una persona viene privata della propria libertà, della propria identità, dell'affetto dei familiari, in uno stato continuo di angoscia dove perfino il cielo lo immagino grigio cupo per questa orribile situazione. Un'immagine e stato d'animo che Guareschi padre avrà sicuramente raccontato al figlio al momento del suo ritorno a casa. 


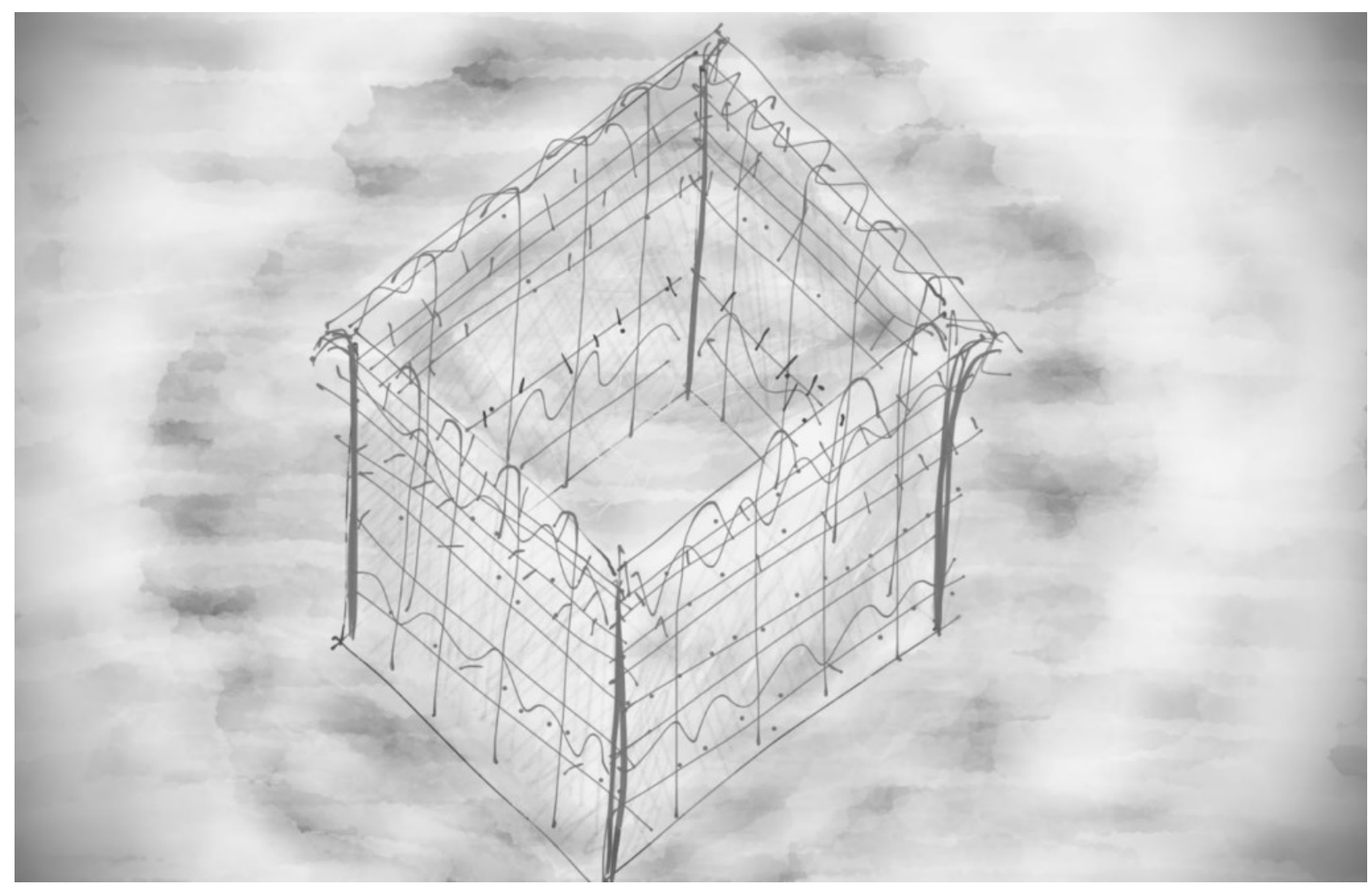

Disegno di Nicolò D.M. 2H

Alessandro, invece ha preferito creare un parallelo tra la quarantena al tempo del Covid-19 e l'assenza di libertà di Guareschi:

La Favola di Natale, mi ispira speranza, forza di volontà e costanza. Ci rendiamo conto dei piccoli elementi che a causa della vita frenetica trascuriamo: la bellezza di accarezzare l'erba fresca o di odorare il profumo di un fiore, o ancora di passare un po' di tempo con i nostri cari, stando insieme a rilassarci. La soddisfazione di fare un bel pranzo con tutti, o le sere a giocare a carte con i nonni. È proprio quando veniamo privati di queste cose, che diamo per scontato, che impariamo ad apprezzare tutto quello che ci circonda, dal primo mattone della nostra casa, alla farfalla fuori la finestra che vola soave e spensierata.

\section{Alessandro P. 2G}

Anche Sofia a cui piace molto scrivere, si è espressa con un paragone con la sua situazione attuale di quarantena:

Nei sogni molto spesso abbiamo quello che desideriamo, ma questo lo fa sembrare anche un incubo. La cosa sconvolgente è quando ti svegli perché svanisce tutto il tuo sogno e ritorni di nuovo alla tua vita.

La fuga in sogno è l'unico sport che ci rimane. Per uscire dalle nostre tristi mura da cui dipendiamo e siamo costretti a stare. Nei sogni non esistono zone della morte e confini. I sogni non hanno bisogno di mangiare perché si nutrono dei nostri piaceri. I sogni non hanno freddo perché si riscaldano con l'Amore. Tutti teniamo sempre con noi la nostra storia (la nostra ricchezza) che ci accompagna anche nei momenti più difficili. Nei sogni non ci si può incontrare perché ci siamo solo noi, e il freddo e la fame oramai diventati $i$ nostri amici di sventura, sempre presenti. Ma $i$ sogni hanno sempre una durata e per questo siamo costretti a ritornare alla triste, malinconica, 
vera, realtà. L'unica cosa che ci rimane è sognare!!! Oramai come Giovannino siamo rinchiusi dentro alla nostra piccola bolla di felicità a pensare a cosa avremmo fatto se non ci sarebbe stato questo ostacolo che impedisce la continuazione della corsa. Lontano dalle sicurezze e incertezze ma vicino alle persone a cui vogliamo bene. La canzone della malinconia è sempre lì che ci attende. La canzone che, che dice la pena a tutti coloro che attendono nelle tristi case.

\section{Sofia A. $2 \mathrm{G}$}

Concludo con la poesia di Benedetta, un'alunna che si esprime spesso attraverso il testo poetico e anche questo lavoro su Guareschi è stato per lei occasione di sperimentazione in versi

\section{LA FAVOLA DI NATALE}

In una notte buia e triste molte persone ancor non si son viste, ma nell'aria più leggera una poesia ancora ci spera: che il padre di Albertino possa tornargli vicino, perciò si mette in cammino partendo di buon mattino. Quanta strada poverina col freddo e con la brina! A cercare tutto il tempo $<$ senza riposar neppure un momento. Va avanti la poesiola $<$ che cammina tutta sola, "Cosa cerchi?" le han chiesto "Cerco un uomo assai onesto, che ha lasciato il suo bambino solo a casa, poverino!"

"Cara mia non puoi entrare o finirai per farti male." Ecco allora che codesta poesiola mesta mesta, Se ne torna da Albertino che piangeva sul cuscino, ma il bambino non si arrende e le scale presto scende. Ha deciso, partirà per cercare del suo papà, con un gruppo squinternato ma giammai disperato, affronta la sua avventura senza mai aver paura.

Nani, funghi, cornacchie e corvi dagli sguardi torvi torvi, passeri, api, un treno e Re magi che spargevano in aria buoni presagi. Quand'ecco apparire il papà di Albertino in cerca di un miglior destino, 
eccolo finalmente, è ormai vicino

ha avuto senso il nostro cammino!

Baci, abbracci e tanto calore

fanno il Natale nel loro cuore.

Benedetta E. 2 G

\section{Bibliografia}

- G. Conti, Giovannino Guareschi. Biografia di uno scrittore, Rizzoli, Milano, 2008.

- G. Frigerio, Noi dei Lager. Testimonianze di militari italiani internati nei campi nazisti 1943-1945, Edizioni Paoline, Milano, 2008.

- G. Guareschi, Il grande diario. Giovannino cronista dei Lager (1943-1945), Rizzoli, Milano, 2008.

- G. Guareschi, La favola di Natale. Con illustrazioni dell'autore e di Arturo Coppola, Edizioni Riunite, Milano, 1946.

- G. Hammermann, Gli internati militari italiani in Germania 1943-1945, Il Mulino, Bologna, 2004.

- $\quad$ S. Pascale, Come stelle nel cielo. In viaggio tra i Lager, Ciesse Edizioni, Padova, 2017.

- S. Pascale, Una candela illumina il Lager, Ciesse Edizioni, Padova, 2018.

- S. Pascale, Fiori dal Lager. Antologia di Internati Militari Italiani" Ciesse Edizioni, Padova, 2019.

- A. Pellai, V. Rinaldin e B. Tamburini, Educazione tra pari. Manuale teorico-pratico di empowered peer education, Erikson, Trento, 2002.

- G. Schreiber, I Militari Italiani Internati nei campi di concentramento del Terzo Reich (1943-1945), SME, Roma, 1997.

- G. Tacconi, La didattica al lavoro. Analisi delle pratiche educative nell'istruzione e formazione professionale, Franco Angeli, Milano, 2013.

- A. Wieviorka, L'era del testimone, Cortina, Milano, 1999.

\section{Note}

[1] Per approfondire l'argomento vedi il prezioso volume G. Schreiber, I Militari Italiani Internati nei campi di concentramento del Terzo Reich (1943-1945), SME, Roma 1997. Per la situazione di vita degli IMI negli Stalag o Oflag vedi G. Hammermann, Gli internati militari italiani in Germania 1943-1945, Il Mulino, Bologna 2004.

[2] La prima stampa dell'opera ristampata di recente, G. Guareschi, La favola di Natale. Con illustrazioni dell'autore e di Arturo Coppola, Edizioni Riunite, Milano, 1946.

[3] Su Guareschi può essere utile questo volume G. Conti, Giovannino Guareschi. Biografia di uno scrittore, Rizzoli, Milano, 2008.

[4] Significativi sull'argomento i seguenti volumi: A. Pellai, V. Rinaldin, B. Tamburini, Educazione tra pari. Manuale teorico-pratico di empowered peer education, Erikson, Trento, 2002; G. Tacconi, La didattica al lavoro. Analisi delle pratiche educative nell'istruzione e formazione professionale, Franco Angeli, Milano, 2013.

[5] A. Wieviorka A., L'era del testimone, Cortina, Milano, 1999, pag. 36. 
